\title{
Ergänzendes Abkürzungsverzeichnis
}

\author{
einschließlich ausgewählter abgekürzt zitierter Literatur"
}

\author{
$\mathrm{aA}$ \\ $\mathrm{aaO}$ \\ $\mathrm{ABl}$ \\ ABlEG, ABlEU
}

Abs

AcP

ADHGB

ADS

$\mathrm{aE}$

$\mathrm{aF}$

AG

AG-S

AGB

AktG

AktG 1937

Aktienrechtsnovelle 2016

Aktionärsrechterichtlinie

AktR

allg

allgM

Alt

aM

Amtl Begr anderer Ansicht

am angegebenen Ort

Amtsblatt

Amtsblatt der Europäischen Gemeinschaften, der Europäischen Union (Nummer, Seite, Datum)

Absatz

Archiv für die civilistische Praxis (Band, Jahr, Seite)

Allgemeines Deutsches Handelsgesetzbuch

Adler, Düring, Schmaltz, Rechnungslegung und Prüfung der Unternehmen, 6. Auflage $1995 \mathrm{ff}$

am Ende

alte Fassung

Amtsgericht; Aktiengesellschaft(en); Die Aktiengesellschaft, Zeitschrift für das gesamte Aktienwesen (Jahr, Seite)

Die Aktiengesellschaft, Zeitschrift für das gesamte Aktienwesen, Sonderheft (Jahr, Seite)

Allgemeine Geschäftsbedingungen

Aktiengesetz v 6.9.1965 (BGBl I 1089; BGBl III/

FNA 4121-1)

Gesetz über Aktiengesellschaften und Kommanditgesellschaften auf Aktien (Aktiengesetz) v 30.1.1937 (RGBl I 107), nunmehr AktG 1965 (AktG)

Gesetz zur Änderung des Aktiengesetzes v 22. 12. 2015 (BGBl I, 2565)

Richtlinie 2007/36/EG des Europäischen Parlaments und des Rates vom 11. Juli 2007 über die Ausübung bestimmter Rechte von Aktionären in börsennotierten Gesellschaften (ABl EU Nr L 184 v 14. 7. 2007, S 17) Aktienrecht

allgemein

allgemeine Meinung

Alternative anderer Meinung

Amtliche Begründung

* Ergänzte und aktualisierte Kurzfassung des im ersten Band abgedruckten allgemeinen Abkürzungsverzeichnisses. Die abgekürzt zitierte Literatur wird in der aktuellen Auflage angegeben. Im Einzelfall kann in der Kommentierung auf Vorauflagen Bezug genommen worden sein. Insoweit wird auf die hochgestellte Ziffer hinter selbständigen Werken verwiesen, welche die benutzte Auflage bezeichnet. 
Angerer/Geibel/Süßmann

AnSVG

Anm

AnwKomm

AP-VO

AR

AReG

ARUG

ARUG II

ArbGG

ArbHdbHV

ARHdb

Art

Assmann/Schneider/Mülbert

Aufl

AuR

BaFin

BAG

BAGE

BAKred

Bank-Betrieb

Baumbach/Hopt
Wertpapiererwerbs- und Übernahmegesetz, Kommentar, 3. Auflage 2017; bis zur 2. Auflage $2008 \mathrm{Geibel} / \mathrm{Süß-}$ mann.

Gesetz zur Verbesserung des Anlegerschutzes (Anlegerschutzverbesserungsgesetz - AnSVG) v 28.10.2004 (BGBl I 2630; BGBl III/FNA 4110-4-1)

Anmerkung

Anwaltkommentar Aktienrecht, hrsg v Heidel, 1. Aufl. 2003; 5. Auflage 2019, Nomoskommentar Aktienrecht und Kapitalmarktrecht (s auch Heidel)

Verordnung (EU) Nr 537/2014 des Europäischen Parlaments und des Rates vom 16. April 2014 über spezifische Anforderungen an die Abschlussprüfung bei Unternehmen von öffentlichem Interesse und zur Aufhebung des Beschlusses 2005/909/EG der Kommission (Abschlussprüfungs-Verordnung), (ABl EU Nr L 158 v 27. 5. 2014, S 77)

Aufsichtsrat

Gesetz zur Umsetzung der prüfungsbezogenen Regelungen der Richtlinie 2014/56/EU sowie zur Ausführung der entsprechenden Vorgaben der Verordnung (EU) Nr 537/ 2014 im Hinblick auf die Abschlussprüfung bei Unternehmen von öffentlichem Interesse (Abschlussprüfungsreformgesetz - AReG) v 10. 5. 2016 (BGBl I, 1142)

Gesetz zur Umsetzung der Aktionärsrechterichtlinie (ARUG) idF v 30.7.2009 (BGBl I 2479)

Gesetz zur Umsetzung der zweiten Aktionärsrechterichtlinie (ARUG II) v 12. 12. 2019 (BGBl I, S 2637) Arbeitsgerichtsgesetz idF v 2.7.1979 (BGBl I 853, ber 1036; BGBl III/FNA 320-1)

Arbeitshandbuch für die Hauptversammlung, hrsg v Reichert, begr v Semler, Volhard, 5. Auflage 2021

Arbeitshandbuch für Aufsichtsratsmitglieder, hrsg v Semler, von Schenck, 4. Auflage 2013 (s auch Semler) Volhard)

Artikel

Wertpapierhandelsgesetz, Kommentar, 7. Auflage 2019; bis zur 6. Auflage 2011 Assmann/Schneider

Auflage

Arbeit und Recht (Jahr, Seite)

Bundesanstalt für Finanzdienstleistungsaufsicht, durch FinDAG ab 1.5.2002, zuvor BAKred, BAV und BAWe Bundesarbeitsgericht Entscheidungen des Bundesarbeitsgerichts (Band, Seite)

Bundesaufsichtsamt für das Kreditwesen, seit 1.5.2002 BaFin

Bank-Betrieb, seit 1977 Die Bank (Jahr und Seite) Handelsgesetzbuch, 39. Auflage 2020 
Ergänzendes Abkürzungsverzeichnis

\author{
Baumbach/Hueck \\ Baumbach/Hueck GmbHG \\ Baums
}

Baums/Thoma

BAV

BAWe

BayObLG

BayObLGZ

$\mathrm{BB}$

$\mathrm{Bd}$, Bde

BeckBil-Komm

BeckHdbAG

Begr, begr

Begr RegE

Beil

Bek

Beschl

BetrVG

$\mathrm{BFH}$

BFHE

BFuP

BGB

BGBl I, II, III

$\mathrm{BGH}$

BGHSt

BGHVGrS

BGHZ

BilKoG

BilMoG

BilReG
Aktiengesetz, 13. Auflage 1968

GmbH-Gesetz, 22. Auflage 2019

Bericht der Regierungskommission Corporate Governance, 2001

WpÜG, Kommentar zum Wertpapiererwerbs- und Übernahmegesetz, Loseblatt, $2004 \mathrm{ff}$

Bundesaufsichtsamt für das Versicherungswesen, seit 1.5.2002 BaFin

Bundesaufsichtsamt für den Wertpapierhandel, seit 1.5.2002 BaFin

Bayerisches Oberstes Landesgericht (aufgelöst seit 1.7.2006)

Entscheidungen des Bayerischen Obersten Landesgerichts in Zivilsachen (Jahr, Seite)

Betriebs-Berater (Jahr, Seite)

Band, Bände

Beck'scher Bilanz-Kommentar, hrsg v Ellrott, 12. Auflage 2020

Beck'sches Handbuch der AG, hrsg v Müller (Welf), Rödder, 3. Auflage 2018

Begründung, begründet

Begründung Regierungsentwurf

Beilage

Bekanntmachung

Beschluss

Betriebsverfassungsgesetz idF v 25.9.2001 (BGBl I 2518; BGBl III/FNA 801-7)

Bundesfinanzhof

Sammlung der Entscheidungen des Bundesfinanzhofs

(Band, Seite)

Betriebswirtschaftliche Forschung und Praxis (Jahr, Seite)

Bürgerliches Gesetzbuch v 18.8.1896 (RGBl 195) idF

v 2.1.2002 (BGBl I 42, ber 2909 und 2003 I 738;

BGBl III/FNA 400-2)

Bundesgesetzblatt Teil I, II und III

Bundesgerichtshof

Entscheidungen des Bundesgerichtshofes in Strafsachen

(Band, Seite)

Bundesgerichtshof, Vereinigter Großer Senat

Entscheidungen des Bundesgerichtshofes in Zivilsachen

(Band, Seite)

Gesetz zur Kontrolle von Unternehmensabschlüssen

(Bilanzkontrollgesetz - BilKoG) v 15.12.2004 (BGBl I 3408)

Gesetz zur Modernisierung des Bilanzrechts (Bilanzrechtsmodernisierungsgesetz - BilMoG) v 25.5.2005

(BGBl I 1102)

Gesetz zur Einführung internationaler Rechnungslegungsstandards und zur Sicherung der Qualität der 
BilRUG

BiRiLiG

BKR

Böckli

BörsG

Bonner HdR

BR

BRD

BRDrucks

BReg

Brodmann

BSG

BSGE

Bsp

BStBl

BT

BTDrucks

BVerfG

BVerfGE

BVerwG

BVerwGE

bzgl

bzw

ca

CEO

c.i.c.

Combined Code

Company Law Action Plan
Abschlussprüfung (Bilanzrechtsreformgesetz - BilReg) v 4.12.2004 (BGBl I 3166)

Gesetz zur Umsetzung der Richtlinie 2013/34/EU des Europäischen Parlaments und des Rates vom 26. Juni 2013 über den Jahresabschluss, den konsolidierten Abschluss und damit verbundene Berichte von Unternehmen bestimmter Rechtsformen und zur Änderung der Richtlinie 2006/43/EG des Europäischen Parlaments und des Rates und zur Aufhebung der Richtlinien 78/660/ EWG und 83/349/EWG des Rates vom 17. Juli 2015 (BGBl I 1245)

Gesetz zur Durchführung der Vierten, Siebenten und Achten Richtlinie des Rates der Europäischen Gemeinschaften zur Koordinierung des Gesellschaftsrechts (Bilanzrichtlinien-Gesetz - BiRiLiG) v 19.12.1985 (BGB1 I 2355)

Zeitschrift für Bank- und Kapitalmarktrecht (Jahr, Seite) Schweizer Aktienrecht, 4. Auflage, Zürich 2009

Börsengesetz (BörsG) 16.7.2007 (BGBl 1330, 1351; BGBl III/FNA 4110-10)

Bonner Handbuch der Rechnungslegung, hrsg v Hofbauer, Kupsch, Scherrer, Grewe, Loseblatt, 1986 ff Bundesrat

Bundesrepublik Deutschland

Bundesrats-Drucksache

Bundesregierung

Aktienrecht, Kommentar, 1928

Bundessozialgericht

Entscheidungen des Bundessozialgerichts

Beispiel

Bundessteuerblatt (Band, Jahr, Seite)

Bundestag

Bundestags-Drucksache

Bundesverfassungsgericht

Entscheidungen des Bundesverfassungsgerichts (Band, Seite)

Bundesverwaltungsgericht

Entscheidungen des Bundesverwaltungsgerichts (Band, Seite)

bezüglich

beziehungsweise

circa

chief executive officer

culpa in contrahendo

The Combined Code on Corporate Governance, July 2003 (Financial Reporting Council, London; Combined Code June 2006 im Konsultationsverfahren der FSA)

Commission of the European Union, Modernising

Company Law and Enhancing Corporate Governance in 


\section{Ergänzendes Abkürzungsverzeichnis}

\section{CorpGov}

COVInsAG

\section{Cozian/Viandier/Deboissy}

CSR-Richtlinie-Umsetzungsgesetz

DAX
DB
DBW
DCGK
ders
dies
DiRUG
Diss
DJT
DNotZ
D\&O-Versicherung
Dörner/Menold/Pfitzer/Oser
Doralt/Nowotny/Kalss
DrittelbG

DStR
DVO
DWiR, DZWir

DZWIR

$\mathrm{E}$

Ebenroth/Boujong/Joost/Strohn

EBOR

ECFR

ECGI

ed(s)

éd the European Union - A Plan to Move Forward, Brussels 21.5.2003, COM(2003) 284 final

Corporate Governance

COVID-19-Insolvenzaussetzungsgesetz, verkündet als

Art. 1 des Gesetzes zur Abmilderung der Folgen der

COVID-19-Pandemie im Zivil-, Insolvenz- und Strafver-

fahrensrecht vom 27. 3.2020 (BGBl I 569)

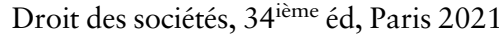

Gesetz zur Stärkung der nichtfinanziellen Berichterstattung der Unternehmen in ihren Lage- und Konzernlageberichten (CSR-Richtlinie-Umsetzungsgesetz) vom 11. 4. 2017 (BGBl I 802)

Deutscher Aktienindex

Der Betrieb (Jahr, Seite)

Die Betriebswirtschaft (Jahr, Seite)

Deutscher Corporate Governance Kodex idF v

26.5.2010, eBAnz AT68 2010 B1, Bek v 2.7.2010

derselbe

dieselbe(n)

Gesetz zur Umsetzung der Digitalisierungsrichtlinie vom 5. 7. 2021 (BGBl I 3338)

Dissertation

Deutscher Juristentag

Deutsche Notar-Zeitschrift, früher Zeitschrift des Deutschen Notarvereins (Jahr, Seite)

directors \& officers liability insurance

Reform des Aktienrechts, der Rechnungslegung und der Prüfung, 2. Auflage 2003

Kommentar zum Aktiengesetz, Wien 2. Auflage 2012

Gesetz über die Drittelbeteiligung der Arbeitnehmer im Aufsichtsrat (Drittelbeteiligungsgesetz - DrittelbG)

v 18.5.2004 (BGBl I 974; BGBl III/FNA 801-14)

Deutsches Steuerrecht (Jahr, Seite)

Durchführungsverordnung

Deutsche Zeitschrift für Wirtschaftsrecht (1991-1998), ab 1999 DZWIR, (Jahr, Seite)

Deutsche Zeitschrift für Wirtschafts- und Insolvenzrecht (Jahr, Seite), vor 1999 DZWir

Entwurf

Handelsgesetzbuch, 4. Auflage 2020 begr v Boujong, Ebenroth, hrsg v Joost, Strohn

European Business Organization Law Review (Band, Jahr, Seite)

European Company and Financial Law Review (Jahr, Seite)

European Corporate Governance Institute, Brüssel

editor(s); edition

édition 


\begin{tabular}{|c|c|}
\hline EG & Einführungsgesetz; Europäische Gemeinschaft(en) \\
\hline EGAktG & $\begin{array}{l}\text { Einführungsgesetz zum Aktiengesetz v } 6.9 .1965 \\
\text { (BGBl I 1185; BGBl III/FNA 4121-2) }\end{array}$ \\
\hline EGBGB & $\begin{array}{l}\text { Einführungsgesetz zum Bürgerlichen Gesetzbuch idF } \\
\text { v 21.9.1994 (BGBl I 2494, ber 1997 I 1061; BGBl III/ } \\
\text { FNA 400-1) }\end{array}$ \\
\hline EGHGB & $\begin{array}{l}\text { Einführungsgesetz zum Handelsgesetzbuche v 10.5.1897 } \\
\text { (RGBl 437; BGBl III/FNA 4101-1) }\end{array}$ \\
\hline EGKomm & Kommission der Europäischen Gemeinschaften \\
\hline EGV & $\begin{array}{l}\text { Vertrag zur Gründung der Europäischen Gemeinschaft } \\
\text { (Amsterdamer Fassung), geändert durch den Vertrag von } \\
\text { Nizza v 26.2.2002 }\end{array}$ \\
\hline Ehricke/Ekkenga/Oechsler & $\begin{array}{l}\text { Wertpapiererwerbs- und Übernahmegesetz, Kommentar, } \\
2003\end{array}$ \\
\hline EHUG & $\begin{array}{l}\text { Gesetz über elektronische Handelsregister und } \\
\text { Genossenschaftsregister sowie das Unternehmensregister } \\
\text { (EHUG) v 10.11.2006 (BGBl I 2553, BGBl III/ } \\
\text { FNA 4100-1) }\end{array}$ \\
\hline Einf & Einführung \\
\hline Einl & Einleitung \\
\hline end & endgültig \\
\hline $\begin{array}{l}\text { Entsch } \\
\text { entspr }\end{array}$ & $\begin{array}{l}\text { Entscheidung } \\
\text { entsprechend }\end{array}$ \\
\hline ErfK & $\begin{array}{l}\text { Erfurter Kommentar zum Arbeitsrecht, begr v Dieterich, } \\
\text { Hanau, Schaub, hrsg v Müller-Glöge, Preis, Schmidt } \\
\text { (Ingrid), 21. Auflage } 2021\end{array}$ \\
\hline ErgG & Ergänzungsgesetz \\
\hline Erman & $\begin{array}{l}\text { Bürgerliches Gesetzbuch, Handkommentar, 16. Auflage } \\
2020\end{array}$ \\
\hline etc & et cetera \\
\hline $\mathrm{EU}$ & $\begin{array}{l}\text { Europäische Union; Vertrag über die Europäische Union } \\
\text { v 7.2.1992 (BGBl II 1251) (s auch EUV) }\end{array}$ \\
\hline EuGH & Gerichtshof der Europäischen Gemeinschaft \\
\hline EuroEG & $\begin{array}{l}\text { Gesetz zur Einführung des Euro (Euro-Einführungs- } \\
\text { gesetz - EuroEG) v 9.6.1998 (BGBl I 1242) }\end{array}$ \\
\hline EUV & $\begin{array}{l}\text { Vertrag über die Europäische Union v 7.2.1992 } \\
\text { (BGBl II 1251) (s auch EU) }\end{array}$ \\
\hline $\begin{array}{l}\text { EuZW } \\
\text { evtl }\end{array}$ & $\begin{array}{l}\text { Europäische Zeitschrift für Wirtschaftsrecht (Jahr, Seite) } \\
\text { eventuell }\end{array}$ \\
\hline EWG & Europäische Wirtschaftsgemeinschaft \\
\hline EWiR & Entscheidungen zum Wirtschaftsrecht (Jahr, Seite) \\
\hline EWIV & Europäische wirtschaftliche Interessenvereinigung \\
\hline $\mathrm{f}, \mathrm{ff}$ & folgende, fortfolgende \\
\hline FamFG & $\begin{array}{l}\text { Gesetz über das Verfahren in Familiensachen und in } \\
\text { den Angelegenheiten der freiwilligen Gerichtsbarkeit idF } \\
\text { v 17.12.2008 (BGBl I 2586, 2587; BGBl 2009 I 1102) }\end{array}$ \\
\hline FASB & Financial Accounting Standards Board \\
\hline Feddersen/Hommelhoff/Schneider & Corporate Governance, 1996 \\
\hline FG & Finanzgericht \\
\hline
\end{tabular}




\section{Ergänzendes Abkürzungsverzeichnis}

FinG
FISG
Forstmoser/Meier
FN
FNA
fragl
FS
Fuchs/Köstler
FüPoG

FüPoG II

Fußn

G

GBl

GbR

Geibel/Süßmann

gem

GenG

Ges

GesR

GesRÄG

GesRRL

GesRZ

Geßler

GG

ggf

$\mathrm{GmbH}$

GmbHG
Finanzgericht (s auch FG)

Gesetz zur Stärkung der Finanzmarktintegrität (Finanz-

marktintegritätsstärkungsgesetz) v 3.6.2021

(BGBl I 1534)

Schweizerisches Aktienrecht, 1996

Fachnachrichten, Institut der Wirtschaftsprüfer in

Deutschland e.V. (Jahr, Seite)

Fundstellennachweis A, Bundesrecht ohne völkerrecht-

liche Verträge (zuvor BGBI III)

fraglich

Festschrift

Handbuch zur Aufsichtsratswahl, 7. Auflage 2021

Gesetz für die gleichberechtigte Teilhabe von Frauen

und Männern an Führungspositionen in der Privatwirt-

schaft und im öffentlichen Dienst vom 24. 4. 2015

(BGBl I 642)

Gesetz zur Ergänzung und Änderung der Regelungen für die gleichberechtigte Teilhabe von Frauen an Führungspositionen in der Privatwirtschaft und im öffentlichen

Dienst vom 7. 8. 2021 (BGBl I 3311)

Fußnote

Gesetz

Gesetzblatt

Gesellschaft bürgerlichen Rechts

s. Angerer/Geibel/Süßmann

gemäß

Gesetz betreffend die Erwerbs- und Wirtschaftsgenossenschaften (Genossenschaftsgesetz) idF v 19.8.1994

(BGBl I 2202; BGBl III/FNA 4125-1)

Gesellschaft

Gesellschaftsrecht

Gesellschaftsrechtsänderungsgesetz (Österreich)

Richtlinie (EU) 2017/1132 des Europäischen Parlaments und des Rates vom 14. 6. 2017 über bestimmte Aspekte des Gesellschaftsrechts (ABl EU Nr L 169 v 30.6.2017, S 46) (Kodifizierter Text) („Gesellschaftsrechtsrichtlinie“)

Der Gesellschafter, Zeitschrift für Gesellschaftsrecht, Wien (Jahr, Seite)

Aktiengesetz, Kommentar, hrsg v Geßler (Ernst), Hefermehl, Eckardt, Kropff, 1973 ff, 2./3. Auflage s Münch-

KommAktG

Grundgesetz für die Bundesrepublik Deutschland v 23.5.1949 (BGBl I 1; BGBl III/FNA 100-1)

gegebenenfalls

Gesellschaft mit beschränkter Haftung

Gesetz betreffend die Gesellschaften mit beschränkter

Haftung v 20.4.1892 (RGBl 477) idF v 20.5.1898

(RGBl I 846; BGBl III/FNA 4123-1) 
GmbHR

(v) Godin/Wilhelmi

Gower/Davies

grds

Großkomm

GrS

GRUR

GS

$\mathrm{GuV}$

GVBl

hA

Haarmann/Riehmer/Schüppen

Habersack/Henssler

Hachenburg

Happ

$\mathrm{Hb}, \mathrm{Hdb}$

HdbAG

Hdb börsennot AG

HdR

Heidel

HeidelbergKomm

Frodermann/Jannott

Heymann

HFA

HGB

High Level Group
GmbH-Rundschau, vorher Rundschau für die $\mathrm{GmbH}$

(Jahr, Seite)

Aktiengesetz, Kommentar, begr v Freiherr von Godin, H. Wilhelmi, 4. Auflage 1971

Gower and Davies' Principles of Modern Company Law, $8^{\text {th }}$ ed, London 2008

grundsätzlich

Aktiengesetz, Großkommentar, begr v Gadow, Heinichen, 1. Auflage 1939, 2. Auflage 1961/65, 3. Auflage $1970 \mathrm{ff}, 4$. Auflage hrsg v Hopt, Wiedemann, $1992 \mathrm{ff}$

Großer Senat

Gewerblicher Rechtsschutz und Urheberrecht (Jahr, Seite)

Gedächtnisschrift

Gewinn- und Verlustrechnung

Gesetz- und Verordnungsblatt

herrschende Ansicht

Öffentliche Übernahmeangebote, Kommentar zum

Wertpapiererwerbs- und Übernahmegesetz, 2002,

3. Auflage Frankfurter Kommentar zum Wertpapier-

erwerbs- und Übernahmegesetz, hrsg v Haarmann,

Schüppen, 2008

Kommentar zum Mitbestimmungsgesetz, Hanau/Ulmer 1981, 2. Auflage Ulmer/Habersack/Henssler MitbestR, 2006; 4. Auflage 2018 Habersack/Henssler

GmbH-Gesetz, Großkommentar, hrsg v Ulmer, 8. Auflage 1992-1997

Aktienrecht, 5. Auflage 2019

Handbuch

Handbuch der Aktiengesellschaft, hrsg v Nirk, Ziemons, Binnewies, Loseblatt, $1999 \mathrm{ff}$

Handbuch börsennotierte AG, hrsg v Marsch-Barner, Schäfer, 4. Auflage 2017

Handbuch der Rechnungslegung, hrsg v Küting, Weber, Loseblatt, $2002 \mathrm{ff}$

5. Auflage 2019 Nomoskommentar (s auch AnwKomm) Heidelberger Kommentar zum Aktiengesetz, hrsg v Bürgers, Körber, Lieder, 5. Auflage 2021

Handbuch des Aktienrechts, 9. Auflage 2017; bis zur

8. Auflage 2009 Henn/Frodermann/Jannott

Handelsgesetzbuch, Kommentar, 2. Auflage hrsg v

Horn, 1995 ff, 3. Auflage 2019 ff.

Hauptfachausschuss des Instituts der Wirtschaftsprüfer in Deutschland e.V.

Handelsgesetzbuch vom 10.5.1897 (RGBl 219; BGBl III/ FNA 4100-1)

High Level Group of Company Law Experts (Winter, chairman, Christensen, Garrido Garcia, Hopt, Rickford, Rossi, Simon), Report of the High Level Group of 


\section{Ergänzendes Abkürzungsverzeichnis}

Hirte Kapitalgesellschaftsrecht

$\mathrm{hL}$

$\mathrm{hM}$

Hoffmann/Lehmann/Weinmann

Hoffmann/Preu

Hommelhoff/Hoptlvon Werder

HommelhofflLutter/Schmidt/

Schön/Ulmer

Hopt Kapitalanlegerschutz

Hopt/Kanda/Roe/Wymeersch/Prigge

Hopt/Voigt
Hopt/Wymeersch
Hopt/Wymeersch
Hopt/Wymeersch/Kanda/Baum
HReg
HRR

Hrsg, hrsg

HRV

Hs

Hucke/Ammann

Hüffer/Koch

HV

IAS

IASB

IASC

idF

idR

IDW

IDW FG

IDW FN

IDW NA
Company Law Experts on Issues Related to Takeover Bids (High Level I), European Commission, Brussels, 10 January 2002; Report of the High Level Group of Company Law Experts on a Modern Regulatory Framework for Company Law in Europe (High Level II), European Commission, Brussels, 4 November 2002 Kapitalgesellschaftsrecht, 8. Auflage 2016 herrschende Lehre herrschende Meinung Mitbestimmungsgesetz, Kommentar, 1978

Der Aufsichtsrat, 5. Auflage 2003

Handbuch Corporate Governance, 2. Auflage 2010

Corporate Governance. Gemeinschaftssymposium der Zeitschriften ZGR/ZHR, ZHR-Beiheft 71, 2002

Der Kapitalanlegerschutz im Recht der Banken, Gesellschafts-, bank- und börsenrechtliche Anforderungen an das Beratungs- und Verwaltungsverhalten der Kreditinstitute, 1975

Comparative Corporate Governance, The State of the Art and Emerging Research, Oxford 1998

Prospekt- und Kapitalmarktinformationshaftung, 2005

Comparative Corporate Governance, Berlin 1997

Capital Markets and Company Law, Oxford 2003

Corporate Governance in Context, Oxford 2005

Handelsregister

Höchstrichterliche Rechtsprechung (1928-1942, zitiert Jahr, Nummer), bis 1927: Die Rechtsprechung, Beilage zur Zeitschrift Juristische Rundschau

Herausgeber, herausgegeben

Verordnung über die Einrichtung und Führung des

Handelsregisters (Handelsregisterverordnung - HRV)

v 12.8.1937 (RMBl 515; DJ 1251; BGBl III/FNA 315-20)

Halbsatz

Der Deutsche Corporate Governance Kodex, 2003

Aktiengesetz, 15. Auflage 2021

Hauptversammlung

International Accounting Standards (seit 1.4.2001 IFRS) International Accounting Standards Board (vor dem 1.4.2001 IASC)

International Accounting Standards Committee (seit 1.4.2001 IASB)

in der Fassung

in der Regel

Institut der Wirtschaftsprüfer in Deutschland e.V.

Fachgutachten des IDW

IDW-Fachnachrichten

Stellungnahmen des Sonderausschusses Neues Aktienrecht und des Hauptfachausschusses des IDW zu Fragen des neuen Aktienrechts 


IDW PS
IDW RH
IDW RS
IDW S
iE
IFRS
insb, insbes
InsO
InvG
IPRax
iÜ
iVm
Jabornegg/Strasser
JBl
Jg
jew
JherJ
JR
JuS
JW

KAGG

Kallmeyer

KapCoRiLiG

$\mathrm{KapMuG}$

$\mathrm{KfH}$

$\mathrm{Kfm}$

KG

$\mathrm{KGaA}$

KGJ

KK
IDW Prüfungsstandard

IDW Rechnungslegungshinweise

IDW Stellungnahme zur Rechnungslegung

IDW Standards

im Ergebnis

International Financial Reporting Standards (vor dem

1.4.2001 IAS)

insbesondere

Insolvenzordnung (InsO) v 5.10.1994 (BGBl I 2866;

BGBl III/FNA 311-13)

Investmentgesetz (InvG) v 15.12.2003 (BGBl I 2676;

BGBl III/FNA 7612-2)

Praxis des internationalen Privat- und Verfahrensrechts

(Jahr, Seite)

im Übrigen

in Verbindung mit

Kommentar zum Aktiengesetz, begr v Schiemer,

5. Auflage, Wien 2011

Justizblatt, Juristische Blätter, Wien (Jahr, Seite)

Jahrgang

jeweils

Jahrbücher für Dogmatik des römischen und deutschen Privatrechts, begr v Jhering, Gerber, später Jherings Jahrbücher für die Dogmatik des Bürgerlichen Rechts (Jahr, Seite)

Juristische Rundschau (Jahr, Seite)

Juristische Schulung (Jahr, Seite)

Juristische Wochenschrift (Jahr, Seite)

Juristenzeitung (Jahr, Seite)

Gesetz über Kapitalanlagegesellschaften (KAGG) idF v 9.9.1998 (BGBl I 2726; BGBl III/FNA 4120-4),

aufgehoben durch InvG

Umwandlungsgesetz, 7. Auflage 2020

Kapitalgesellschaften \& Co.-Richtlinie-Gesetz

v 24. 2. 2000 (BGBl I 154)

Gesetz über Musterverfahren in kapitalmarktrechtlichen Streitigkeiten (Kapitalanleger-Musterverfahrensgesetz KapMuG) idF v 16.8.2005 (BGBl I 2437)

Kammer für Handelssachen

Kaufmann

Kommanditgesellschaft, Kammergericht

Kommanditgesellschaft auf Aktien

Jahrbuch für Entscheidungen des Kammergerichts in

Sachen der freiwilligen Gerichtsbarkeit (Band, Seite)

Kölner Kommentar zum Aktiengesetz, hrsg v Zöllner, 1. Auflage 1970 ff, 2. Auflage 1988 ff, 3. Auflage hrsg v Zöllner, Noack, 2004 ff 


\section{Ergänzendes Abkürzungsverzeichnis}

KK-WpÜG

Klausing

Köstler/Zachert/Müller

Koller/Roth/Morck

$\mathrm{KOM}$

Komm

KonTraG

KostREuroUG

Kraakman/Davies/Hansmann/

Hertig/Hopt/Kanda/Rock

KredAufwErsVO

krit

Kropff AktG

KTS

Kübler/Assmann GesR

KWG

LAG

LG

li $\mathrm{Sp}$

Lit

LS

Lutter/Winter

Lutter/Hommelhoff GmbHG

Lutter Information

Lutter/Krieger/Verse
Kölner Kommentar zum Wertpapiererwerbs- und Übernahmegesetz, hrsg v Hirte, von Bülow, 2. Auflage 2010

Gesetz über Aktiengesellschaften und Kommanditgesellschaften auf Aktien (Aktiengesetz) nebst Einführungsgesetz und „Amtlicher Begründung“ (AktG 1937) Aufsichtsratspraxis, Handbuch für Arbeitnehmervertreter im Aufsichtsrat, 10. Auflage 2013

Handelsgesetzbuch, Kommentar, 9. Auflage 2019

Kommission der Europäischen Gemeinschaften (Dokumente)

Kommentar

Gesetz zur Kontrolle und Transparenz im Unternehmensbereich (KonTraG) v 27.4.1998 (BGBl I 786; BGBl III/FNA 4121)

Gesetz zur Umstellung des Kostenrechts und der Steuerberatergebührenverordnung auf Euro (KostREuroUG) v 27.4.2001 (BGBl I 751)

The Anatomy of Corporate Law. A Comparative and Functional Approach, Oxford 2004

Verordnung über den Ersatz von Aufwendungen der

Kreditinstitute v 17. 6. 2003 (BGBl I 885)

kritisch

Aktiengesetz vom 6.9.1965 und Einführungsgesetz zum Aktiengesetz mit Begründung des Regierungsentwurfs, 1965

Zeitschrift für Insolvenzrecht, Konkurs, Treuhand, Sanierung, (Jahr, Seite)

Gesellschaftsrecht, 6. Auflage 2006, 5. Auflage Kübler, 1998

Gesetz über das Kreditwesen idF v 9.9.1998

(BGBl I 2776; BGBl III/FNA 7610-1)

Landesarbeitsgericht

Landgericht

linke Spalte

Literatur

Leitsatz

Umwandlungsgesetz, Kommentar, 6. Auflage 2019

GmbH-Gesetz, Kommentar, 20. Auflage 2020

Information und Vertraulichkeit im Aufsichtsrat, 3. Auflage 2006

Rechte und Pflichten des Aufsichtsrats, 7. Auflage 2020; bis 6. Auflage 2014 Lutter/Krieger

mit

mit anderen Worten

Monatsschrift für Deutsches Recht (Jahr, Seite)

US-amerikanisches Gesellschaftsrecht, 3. Auflag 2013;

bis 2. Auflage 2006 Merkt/Göthel 
MinG

MitbestBeiG

MitbestErgG

MitbestG

Mitt

MoMiG

Montan-MitbestG

Mülbert Aktiengesellschaft

MünchAnwHdb Aktienrecht

MünchHdbAG

MünchKommAktG

MünchKommBGB

MünchKommHGB

mwN

$\mathrm{MwSt}$

$\mathrm{mWv}$

Nachw

NASDAQ

$\mathrm{NaStraG}$
Ministergesetz

Gesetz zur Beibehaltung der Mitbestimmung beim Austausch von Anteilen und der Einbringung von Unternehmensteilen, die Gesellschaften verschiedener Mitgliedstaaten der Europäischen Union betreffen (Mitbestimmungs-Beibehaltungsgesetz - MitbestBeiG) v 23.8.1994 (BGBl I 2228)

Gesetz zur Ergänzung des Gesetzes über die Mitbestimmung der Arbeitnehmer in den Aufsichtsräten und Vorständen der Unternehmen des Bergbaus und der Eisen und Stahl erzeugenden Industrie v 7.8.1956 (BGBl I 707; BGBl III/FNA 801-3)

Gesetz über die Mitbestimmung der Arbeitnehmer (Mitbestimmungsgesetz - MitbestG) v 4.5.1976

(BGBl I 1153; BGBl III/FNA 801-8)

Mitteilungen

Gesetz zur Modernisierung des GmbH-Rechts und zur Bekämpfung von Missbräuchen (MoMiG) v 23.10.2008 (BGBl I 2026)

Gesetz über die Mitbestimmung der Arbeitnehmer in den Aufsichtsräten und Vorständen der Unternehmen des Bergbaus und der Eisen und Stahl erzeugenden Industrie v 21.5.1951 (BGBl I 347; BGBl III/FNA 801-2)

Aktiengesellschaft, Unternehmensgruppe und Kapitalmarkt. Die Aktionärsgruppe bei Bildung und Umbildung einer Unternehmensgruppe zwischen Verbands- und Anlegerschutzrecht, 2. Auflage 1996

Münchener Anwaltshandbuch Aktienrecht, hrsg v Schüppen, Schaub, 3. Auflage 2018

Münchener Handbuch des Gesellschaftsrechts Band 4: Aktiengesellschaft, hrsg v Hoffmann-Becking, 4. Auflage 2015

Münchener Kommentar zum Aktiengesetz, hrsg v Kropff, Semler, 5. Auflage 2019 ff, hrsg v Goette, Habersack, 4. Auflage 2016 ff; 1 . Auflage s Geßler Münchener Kommentar zum Bürgerlichen Gesetzbuch, hrsg v Rebmann, Säcker, Rixecker, 7. Auflage 2018 ff; Bände 1, 3 und 8 in 6. Auflage $2012 \mathrm{ff}$

Münchener Kommentar zum Handelsgesetzbuch, hrsg v K. Schmidt, 4. Auflage 2016 ff.

mit weiteren Nachweisen

Mehrwertsteuer

mit Wirkung vom

Nachweis

National Association of Securities Dealers Automated Quotations (USA)

Gesetz zur Namensaktie und zur Erleichterung der Stimmrechtsausübung (Namensaktiengesetz - NaStraG) v 18.1.2001 (BGBl I 123; BGBl III/FNA 4121-1) 


\section{Ergänzendes Abkürzungsverzeichnis}

$\mathrm{nF}$
$\mathrm{NJ}$
$\mathrm{NJW}$
$\mathrm{NJW}-\mathrm{RR}$
$\mathrm{Nr}(\mathrm{n})$
$\mathrm{NYSE}$
$\mathrm{NZA}$
$\mathrm{NZG}$
Obermüller/Werner/Winden

OECD

Österr OGH
OFD
OGH
OGHZ

OHG

OLG

OLGZ

Palandt

Peltzer

Pfitzer/Oser

PotthoffiTrescher

PublG

$\mathrm{pVV}$

RabelsZ

RAG

Raiser/Veil/Jacobs

Raiser/Veil Kapitalgesellschaften RBegrG

$\mathrm{Rdn}$

RdA

RdW

Recht neue Fassung

Neue Justiz (Jahr, Seite)

Neue Juristische Wochenschrift (Jahr, Seite)

NJW-Rechtsprechungs-Report Zivilrecht (Jahr, Seite)

Nummer(n)

New York Stock Exchange

Neue Zeitschrift für Arbeits- und Sozialrecht, seit 1992

Neue Zeitschrift für Arbeitsrecht (Jahr, Seite)

Neue Zeitschrift für Gesellschaftsrecht (Jahr, Seite)

Die Hauptversammlung der Aktiengesellschaft, 5. Auflage 2011, bearb v Butzke

Organisation for Economic Cooperation and Development

Österreichischer Oberster Gerichtshof

Oberfinanzdirektion (Jahr, Seite)

Oberster Gerichtshof für die Britische Zone

Entscheidungen des Obersten Gerichtshofes für die Britische Zone in Zivilsachen (1949/50, zitiert Band, Seite)

Offene Handelsgesellschaft

Oberlandesgericht

Entscheidungen der Oberlandesgerichte in Zivilsachen (Jahr, Seite)

Bürgerliches Gesetzbuch, 80. Auflage 2021

Deutsche Corporate Governance, 2. Auflage 2004

Deutscher Corporate Governance Kodex, 2003,

2. Auflage 2005 hrsg v Pfitzer, Oser, Orth

Das Aufsichtsratsmitglied, 6. Auflage 2003, bearb v

Theisen

Gesetz über die Rechnungslegung von bestimmten

Unternehmen und Konzernen (Publizitätsgesetz - PublG)

v 15.8.1969 (BGBl I 1189, ber 1970 I 1113; BGBl III/

FNA 4120-7)

positive Vertragsverletzung

Rabels Zeitschrift für ausländisches und internationales Privatrecht (Band, Jahr, Seite)

Reichsarbeitsgericht, Entscheidungen des Reichsarbeitsgerichts (Band, Seite)

Mitbestimmungsgesetz, Kommentar, 7. Auflage 2020

Recht der Kapitalgesellschaften, 6. Auflage 2015

Gesetz zur Begrenzung der mit Finanzinvestitionen

verbundenen Risiken (Risikobegrenzungsgesetz) $\mathrm{v}$ 12.8.2008 (BGBl I 1666)

Randnummer(n) (s auch Rn)

Recht der Arbeit (Jahr, Seite)

Recht der Wirtschaft, Wien (Jahr, Seite)

Das Recht (Jahr, Nummer der Entscheidung; bei Aufsätzen: Jahr, Seite) 
RefE
RegE
re Sp
Restrukturierungsgesetz

RG

RGBl I, II

RGZ

Ringleb/Kremer/Lutter/vonWerder

Ritter

RIW

RJA

RL

$\mathrm{Rn}$

ROHG

ROHGE

Röhricht/Grafvon Westphalen/Haas

Rowedder/Schmidt-Leithoff

Roth/Altmeppen

Rspr

$\mathrm{S}$

S

SanInsFoG

Schlegelberger/Quassowski

K. Schmidt GesR

Scholz

Schwark/Zimmer

SE

SEAG

SEBG

SEC

SEEG
Referentenentwurf

Regierungsentwurf

rechte Spalte

Gesetz zur Restrukturierung und geordneten Abwicklung von Kreditinstituten, zur Errichtung eines Restrukturierungsfonds für Kreditinstitute und zur Verlängerung der Verjährungsfrist der aktienrechtlichen Organhaftung v. 9. 12. 2010 (BGBl I 1900)

Reichsgericht (Band, Seite)

Reichsgesetzblatt, von 1922-1945 Teil I und Teil II

(Jahr, Seite)

Entscheidungen des Reichsgerichts in Zivilsachen (Band, Seite)

Kommentar zum Deutschen Corporate Governance

Kodex, 4. Auflage 2010

Aktiengesetz, 2. Auflage 1939

Recht der internationalen Wirtschaft (Jahr, Seite)

Entscheidungen in Angelegenheiten der freiwilligen

Gerichtsbarkeit, zusammengestellt vom Reichsjustizamt

(Band, Seite)

Richtlinie

Randnummer(n) (s auch Rdn)

Reichsoberhandelsgericht

Entscheidungen des Reichsoberhandelsgerichts (Band, Seite)

Handelsgesetzbuch, Kommentar, 5. Auflage 2019

GmbHG, Kommentar, 6. Auflage 2017

GmbHG, Kommentar, 10. Auflage 2021

Rechtsprechung

siehe

Seite; Satz

Sanierungs- und Insolvenzrechtsfortentwicklungsgesetz v 22. 12. 2020 (BGBl I 3256)

Aktiengesetz, Kommentar, 3. Auflage 1939

Gesellschaftsrecht, 4. Auflage 2002

Kommentar zum GmbH-Gesetz, 12. Auflage 2018

Kapitalmarktrechts-Kommentar, 5. Auflage 2020

Societas Europaea, Europäische Aktiengesellschaft

Gesetz zur Ausführung der Verordnung (EG) Nr. 2157/

2001 des Rates vom 8. Oktober 2001 über das Statut der

Europäischen Gesellschaft (SE) (SE-Ausführungsgesetz-

SEAG) v 22.12.2004 (BGBl I 3675; BGBl III/FNA 4121-4)

Gesetz über die Beteiligung der Arbeitnehmer in einer

Europäischen Gesellschaft (SE-Beteiligungsgesetz -

SEBG) v 22.12.2004 (BGBl I 3686; BGBl III/

FNA 801-15)

Securities and Exchange Commission (USA)

Gesetz zur Einführung der Europäischen Gesellschaft v

22.12.2004 (BGBl I 3675) 


\section{Ergänzendes Abkürzungsverzeichnis}

Semler

\section{Semler/Volhard}

SeuffArch

SE-VO

Slg

Soergel

$\operatorname{sog}$

SprAuG

Spark

SpruchG

Spruchverfahrensneuordnungsgesetz

Staub

Staudinger

Steinmeyer

StGB

str

st Rspr

StückAG

SZW/RSDA

Teichmann/Koehler

Theisen
Leitung und Überwachung der Aktiengesellschaft, 2. Auflage 1996

Arbeitshandbuch für Aufsichtsratsmitglieder, 3. Auflage 2009 (s auch ARHdb)

Seufferts Archiv für Entscheidungen der obersten Gerichte (Band, Nummer)

Verordnung (EG) Nr. 2157/2001 des Rates über das

Statut der Europäischen Gesellschaft (SE) (ABIEG L 294/

$1 \mathrm{v} 10.11 .2001)$

Sammlung

Kommentar zum Bürgerlichen Gesetzbuch, 13. Auflage $1999 \mathrm{ff}$

sogenannte(r)

Gesetz zur Änderung des Betriebsverfassungsgesetzes, über Sprecherausschüsse der leitenden Angestellten und zur Sicherung der Montan-Mitbestimmung v 20.12.1988 (BGBl I 2312; BGBl III/FNA 801-11)

Die Sparkasse, Zeitschrift des deutschen Sparkassen- und Giroverbandes (Jahr, Seite)

Gesetz über das gesellschaftsrechtliche Spruchverfahren (Spruchverfahrensgesetz), eingeführt durch Art. 1 des Gesetzes zur Neuordnung des gesellschaftsrechtlichen Spruchverfahrens (Spruchverfahrensneuordnungsgesetz) v 12. 6. 2003 (BGBl I 838)

Gesetz zur Neuordnung des gesellschaftsrechtlichen Spruchverfahrens (Spruchverfahrensneuordnungsgesetz) v 12. 6. 2003 (BGBl I 838)

Handelsgesetzbuch, Großkommentar, 4. Auflage 1983 ff, Bände 2, 3, 6 und 7/1 in 5. Auflage 2008 ff, hrsg v Canaris, Habersack, Schäfer, 6. Aufl. 2021 ff, hrsg v Grundmann, Habersack, Schäfer

Kommentar zum Bürgerlichen Gesetzbuch, Neubearbeitung $1999 \mathrm{ff}$

WpÜG, Kommentar zum Wertpapiererwerbs- und Übernahmegesetz, 4. Auflage 2019

Strafgesetzbuch idF v 13.11.1998 (BGBl I 3322;

BGBl III/FNA 450-2)

strittig, streitig

ständige Rechtsprechung

Gesetz über die Zulassung von Stückaktien (Stückaktiengesetz - StückAG) v 25.3.1998 (BGBl I 590; BGBl III/

FNA 4121-1)

Schweizerische Zeitschrift für Wirtschaftsrecht, Revue suisse de droit des affaires (früher SchweizAG, Jahr, Seite)

Aktiengesetz, Kommentar, 3. Auflage 1950

Grundsätze einer ordnungsmäßigen Information des Aufsichtsrats, 3. Auflage 2002, Information und Berichterstattung des Aufsichtsrats, 4. Auflage 2008 
TransPuG

TUG

$\mathrm{u}$

ua

überw

Ulmer/Habersack/Henssler MitbestR

UMAG

UmwG

unstr

unzutr

Urt

USA

US-GAAP

usw

$\mathrm{V}$

VAG

VerfGH

Verh

VersR

VfGH

vgl

$\mathrm{VO}(\mathrm{en})$

Voigt

Voraufl

Vorb, Vorbem

VorstAG
Gesetz zur weiteren Reform des Aktien- und Bilanzrechts, zu Transparenz und Publizität (Transparenz- und Publizitätsgesetz) v 19.7.2002 (BGBl I 2681; BGBl III/ FNA 4121-1)

Gesetz zur Umsetzung der Richtlinie 2004/109/EG des Europäischen Parlaments und des Rates vom 15. Dezember 2004 zur Harmonisierung der Transparenzanforderungen in Bezug auf Informationen über Emittenten, deren Wertpapiere zum Handel auf einem geregelten Markt zugelassen sind, und zur Änderung der Richtlinie 2001/34/EG (Transparenzrichtlinie-Umsetzungsgesetz TUG) v 5.1.2007 (BGBl I 10)

unten

unter anderem; und andere

überwiegend

Mitbestimmungsrecht, Kommentierung des MitbestG, der DrittelbG und der $\mathbb{\$} \$ 34$ bis 38 SEBG,

s. Habersack/Henssler

Gesetz zur Unternehmensintegrität und Modernisierung des Anfechtungsrechts (UMAG) v 22.9.2005 (BGBl I 2802; BGBl III/FNA 4121-1)

Umwandlungsgesetz idF v 28.10.1994 (BGBl I 3210, ber 2005 I 428; BGBl III/FNA 4120-9-2)

unstreitig

unzutreffend

Urteil

United States of America

United States Generally Accepted Accounting

Principles

und so weiter

von; vom

Gesetz über die Beaufsichtigung der Versicherungsunternehmen (Versicherungsaufsichtsgesetz - VAG) idF

v 17.12.1992 (BGBl 1993 I 2; BGBl III/FNA 7631-1)

Verfassungsgerichtshof (s auch VfGH)

Verhandlungen des Deutschen Bundestages (BT), des

Deutschen Juristentages (DJT) usw

Versicherungsrecht, Juristische Rundschau für die

Individualversicherung (Jahr, Seite)

Verfassungsgerichtshof (s auch VerfGH)

vergleiche

Verordnung(en)

Haftung aus Einfluss auf die Aktiengesellschaft, 2004

Vorauflage

Vorbemerkung

Gesetz über die Angemessenheit von Vorstandsvergütungen (VorstAG) idF v 31.7.2009 (BGBl I 2509) 


\section{Ergänzendes Abkürzungsverzeichnis}

VorstOG

\author{
WiB \\ Wiedemann Gesellschaftsrecht \\ Widmann/Mayer
}

wistra

Wlotzke/Wißmann/Koberskil

Kleinsorge

WM

WP

WPg

WpHG

WPK

WpÜG

WuB

$\mathrm{zB}$

ZBB

ZEuP

ZfA

$\mathrm{ZfB}$

$\mathrm{ZfbF}$

ZfRV

ZGR

ZHR

ZIP

ZRP

ZVglRWiss

ZZP

Zweite Aktionärsrechterichtlinie
Gesetz über die Offenlegung von Vorstandsvergütungen (Vorstandsvergütungs-Offenlegungsgesetz - VorstOG) v 3.8.2005 (BGBl I 2267)

Wirtschaftsrechtliche Beratung (Jahr, Seite)

Gesellschaftsrecht, Band I 1980, Band II 2004

Umwandlungsrecht, Kommentar, hrsg v Widmann, Mayer, Loseblatt, 182. Aktualisierung 2020

Zeitschrift für Wirtschafts- und Steuerstrafrecht (Jahr, Seite)

Mitbestimmungsrecht, Kommentar, 4. Auflage 2011

Wertpapier-Mitteilungen (Jahr, Seite)

Das Wertpapier (Jahr, Seite)

Die Wirtschaftsprüfung (Jahr, Seite)

Gesetz über den Wertpapierhandel (Wertpapier-

handelsgesetz - WpHG) idF v 9.9.1998 (BGBl I 2708;

BGBl III/FNA 4110-4)

Wirtschaftsprüferkammer

Wertpapiererwerbs- und Übernahmegesetz (WpÜG) v 20.12.2001 (BGBl I 3822; BGBl III/FNA 4110-7)

Entscheidungssammlung zum Wirtschafts- und

Bankrecht

zum Beispiel

Zeitschrift für Bankrecht und Bankwirtschaft (Jahr, Seite)

Zeitschrift für Europäisches Privatrecht (Jahr, Seite)

Zeitschrift für Arbeitsrecht (Band, Jahr, Seite)

Zeitschrift für Betriebswirtschaft (Band, Jahr, Seite)

Schmalenbachs Zeitschrift für betriebswirtschaftliche

Forschung (Band, Jahr, Seite)

Zeitschrift für Rechtsvergleichung, Internationales Pri-

vatrecht und Europarecht (Jahr, Seite)

Zeitschrift für Unternehmens- und Gesellschaftsrecht

(Jahr, Seite)

Zeitschrift für das gesamte Handelsrecht und Wirt-

schaftsrecht (Band, Jahr, Seite)

Zeitschrift für Wirtschaftsrecht (Jahr, Seite)

Zeitschrift für Rechtspolitik (Jahr, Seite)

Zeitschrift für Vergleichende Rechtswissenschaft (Band, Jahr, Seite)

Zeitschrift für Zivilprozess (Band, Jahr, Seite)

Richtlinie (EU) 2017/828 des Europäischen Parlaments und des Rates vom 17. Mai 2017 zur Änderung der Richtlinie 2007/36/EG im Hinblick auf die Förderung der langfristigen Mitwirkung der Aktionäre (ABl EU

Nr L 132 v 20.5.2017, S 1) 
